\title{
A High Efficiency Doherty Power Amplifier for TV Band Applications
}

\author{
Mohamad Y. Abou-Shahine, Youssef Nasser, Karim Y. Kabalan \\ Electrical and Computer Engineering Department, American University of Beirut, Beirut, Lebanon \\ Email: mya26@aub.edu.lb, yn10@aub.edu.lb, kabalan@aub.edu.lb
}

Received 3 November 2015; accepted 6 December 2015; published 9 December 2015

Copyright (C) 2015 by authors and Scientific Research Publishing Inc.

This work is licensed under the Creative Commons Attribution International License (CC BY). http://creativecommons.org/licenses/by/4.0/

c) (i) Open Access

\begin{abstract}
This paper presents a high efficiency Doherty power amplifier suitable for TV band applications. A class $A B$ power amplifier is firstly implemented using a commercial GaN HEMT from Cree Incorporation, achieving a high power-added-efficiency of $77.78 \%$ and a $40.593 \mathrm{dBm}$ output power with an associated gain of $\mathbf{2 1 . 6 5} \mathrm{dB}$. The Doherty amplifier has then been designed following the previous class $A B$ scheme for the main amplifier and a class $C$ scheme for the peak one. This amplifier attained a high power-added-efficiency of $81.94 \%$, a $42.77 \mathrm{dBm}$ output power, an associated gain of $21.32 \mathrm{~dB}$, and an operating frequency bandwidth between 550 and $1000 \mathrm{MHz}$ (58.06\% fractional bandwidth) which made it suitable for TV band applications.
\end{abstract}

\section{Keywords}

Power Amplifier, Class AB, Doherty Power Amplifier, Efficiency, TV Band

\section{Introduction}

Recently, the design of RF front-ends in wireless communication for cognitive radio applications has become a hot research area. Highly efficient and linear power amplifier is a key component in these systems. However, the main problems of the design of power amplifier exist in their linearity region and their gain especially in multicarrier systems (such as orthogonal frequency division multiplexing-OFDM) which are widely used in current wireless communication. Indeed, these systems present high peak-to-average power ratio (PAPR) caused by the large number of independent subcarriers with random phase and amplitude added together at the input of the amplifier which makes the latter impractical to work next to its saturation point [1]. Therefore, a power amplifier with high linearity and efficiency is great of importance. The simplest method is to back-off signals from the saturation region to the linear region at the cost of power efficiency. Another way may use predistortion methods or elimination and restoration techniques. However, these techniques which need additional components 
result in an increase in cost, size, and power dissipation. In order to solve these problems, a Doherty Power Amplifier (DPA) is the most promising candidate with high efficiency in power and bandwidth [2]-[8].

The Doherty amplifier operation can be achieved using two cells with a class-AB biased carrier amplifier cell and a class-C biased peak amplifier cell with respective input matching network and output matching network. The Doherty power Amplifier (DPA) operating principle is based on modulating the load of the active (carrier) device by using a second active peak device. The two power amplifiers are connected at the output through a quarter-wave transmission line to exploit the active load modulation concept performed by the peak amplifier on the main carrier one. This Doherty amplifier has high linearity and efficiency across the wideband signal.

In practice, Doherty amplifiers are the best candidates that can provide higher efficiency among all type of amplifiers. They are widely used for many applications where RF power amplifier efficiency is important. The efficiency of the Doherty amplifier can be improved if the efficiencies of the two amplifiers can be properly increased [4].

The Doherty technique is usually adopted to design power amplifiers for wireless systems and, in particular, in base stations, working in L-S-C Band for applications such as WiMAX, WLAN, cellular network, and others. Drain efficiencies up to 70\% have been demonstrated for output powers between $5 \mathrm{~W}$ and $10 \mathrm{~W}$ [9]-[11], whereas drain efficiency up to $50 \%$ has been demonstrated for $250 \mathrm{~W}$ output power [12].

However, a well-known disadvantage of the DPA is its typical narrow bandwidth performance. Although significant improvements in bandwidth have been reported recently [13]-[17], the DPA is still fundamentally bandwidth limited by the quarter wave impedance inverter needed to obtain proper load modulation. This results in substantial frequency dependence at the back-off power region where the Power Amplifier (PA) is operating most of the time, and thereby narrow band performance for realistic signals.

In this paper, a class $\mathrm{AB}$ power amplifier and a high efficiency Doherty one with operating frequency at 800 $\mathrm{MHz}$ were developed and verified for the suitability for TV band applications. In comparison with previously designed power amplifiers, the proposed Doherty power amplifier attains higher efficiency and wider bandwidth. The proposed Doherty power amplifier reaches a power added efficiency of $81.94 \%$ with a $58.06 \%$ fractional bandwidth, so that it can be used as a high efficiency power amplifier for the TV band from 550 to $1000 \mathrm{MHz}$. The geometry, the design guidelines, and the results of the proposed amplifiers are presented. In addition, the characteristics of the designed amplifiers are investigated via Agilent Advanced Design System (ADS) software and verified using NI AWR Design Environment software.

\section{Design, Simulation, and Results}

\subsection{Class AB Power Amplifier}

The schematic of this proposed class AB amplifier is shown in Figure 1. The amplifier is simulated in both software, ADS and AWR. The active device employed in the AB amplifier is the CGH40010 from Cree Inc., a GaN HEMT with a typical output power of $10 \mathrm{~W}$ at the suggested drain bias voltage of $28 \mathrm{~V}$. This transistor offers high efficiency, high gain, and wideband capabilities. The amplifier circuit was simulated with a constant drain supply voltage Vsup $=28 \mathrm{~V}$ and the gate bias voltage $\mathrm{Vg}=-2.1 \mathrm{~V}$ which represents a bias condition of a conventional class $\mathrm{AB}$ amplifier. The substrate parameters were set according to Rogers RO3203 substrate with dielectric constant $\varepsilon_{r}=3.02$ and thickness $\mathrm{h}=1.6 \mathrm{~mm}$, on which the amplifier is designed.

To verify the accurate representation of the Cree CGH40010 model, the signal scattering parameters generated from ADS for the frequency $800 \mathrm{MHz}$ were compared to the ones given in the datasheet. Close agreement, as presented in Table 1, is obtained.

After carrying out the stability test of this amplifier, it is found that the device is potentially unstable in the frequency range of interest. Indeed, the stability parameters, $\Delta$, and the rollet factor $k$ are calculated and found to be:

$$
\begin{aligned}
& \Delta=0.092 \angle-8.6^{\circ} \\
& k=0.163
\end{aligned}
$$

This means that the device is potentially unstable. Stabilization methods were performed and series resistances were added to the input and the output of the transistor as shown in Figure 1. The input resistance is 3.5 Ohms and the output resistance is 32.5 Ohms. After adding these resistances, the stability parameters were recalculated and found to be: 


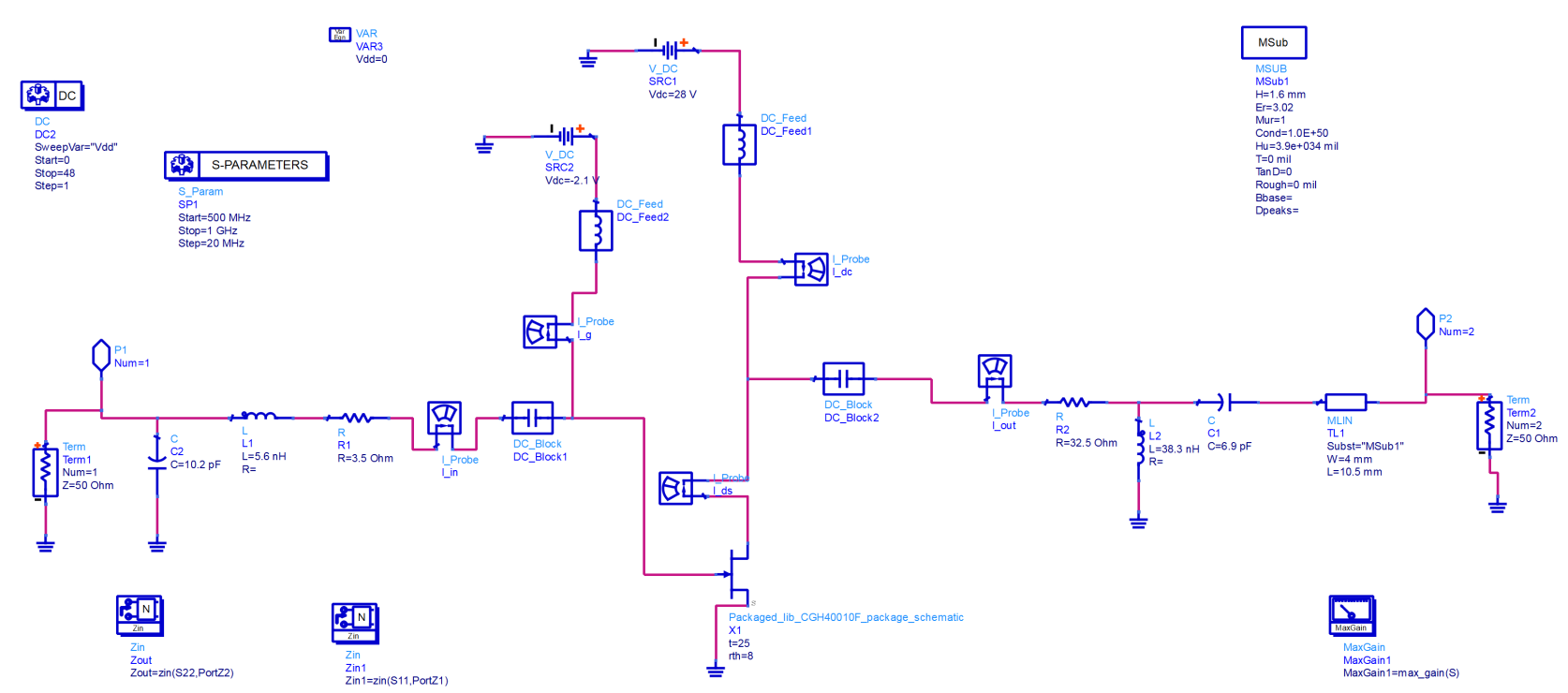

(a)

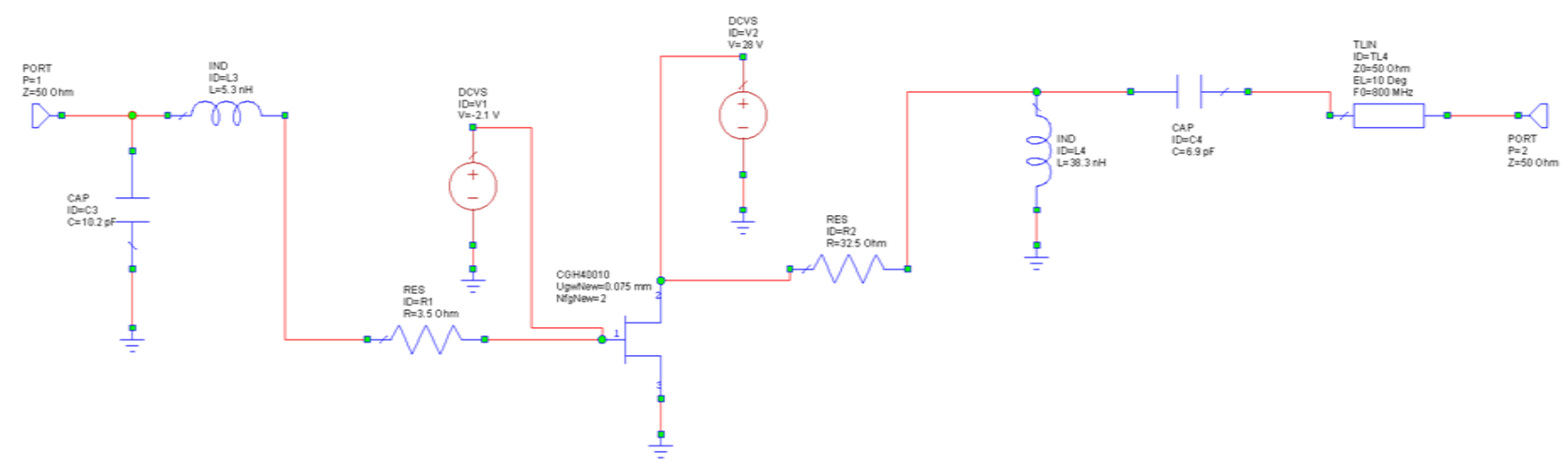

(b)

Figure 1. Schematic of the AB power amplifier with input and output stability resistors and matching circuits. (a) In ADS; (b) In AWR.

Table 1. S-parameters of the GaN transistor at $800 \mathrm{MHz}$.

\begin{tabular}{cccc}
\hline & S11 & S12 & S21 \\
\hline Simulation & $0.911\left\llcorner-154^{\circ}\right.$ & $0.023\left\llcorner 3.54^{\circ}\right.$ & $11.933\left\llcorner 89.38^{\circ}\right.$ \\
Datasheet & $0.894\left\llcorner-146.96^{\circ}\right.$ & $0.029\left\llcorner 6.84^{\circ}\right.$ & $11.58\left\llcorner 91.78^{\circ}\right.$ \\
\hline
\end{tabular}

$$
\begin{aligned}
& \Delta=0.162 \angle-146.4^{\circ} \\
& k=1.57
\end{aligned}
$$

After adding the stability resistances, the signal scattering parameters at $800 \mathrm{MHz}$ had changed. The scattering parameters were simulated and found to be (at $800 \mathrm{MHz}$ ):

$$
\begin{aligned}
& \mathrm{S} 11=0.772 \angle-157.51^{\circ} \\
& \mathrm{S} 12=0.016 \angle-0.034^{\circ} \\
& \mathrm{S} 21=8.057 \angle 85.81^{\circ} \\
& \mathrm{S} 22=0.17 \angle-39.773^{\circ}
\end{aligned}
$$

In order to maintain maximum power transfer, we need to match the impedance of the load to that of the source, so matching circuits are designed at the input and the output of the transistor. These circuits are accomplished by using passive networks and microstrip transmission lines as shown in Figure 2. 
After completing the stability and the matching of the amplifier, the final signal scattering parameters at 800 $\mathrm{MHz}$ were simulated and found to be:

$$
\begin{aligned}
& \mathrm{S} 11=0.056 \angle-119.8^{\circ} \\
& \mathrm{S} 12=0.024 \angle-72.2^{\circ} \\
& \mathrm{S} 21=12.073 \angle 13.6^{\circ} \\
& \mathrm{S} 22=0.438 \angle-89.4^{\circ}
\end{aligned}
$$

By feeding the amplifier with an optimum $800 \mathrm{MHz}, 18.943 \mathrm{dBm}$ input power, the output power of the amplifier circuit was found to be $40.593 \mathrm{dBm}$ and the gain is $21.65 \mathrm{~dB}$. The DC power is simulated and found to be:

$$
\mathrm{Pdc}=28 \times 0.522=14.62 \mathrm{~W}
$$

Thus, the power added efficiency (PAE) is computed to be around $77.78 \%$. All the results are summarized in Table 2.

The amplifier efficiency depends on the DC supply voltage and respectively on the input power. Figure 3 shows the output power sweep curve as well as the corresponding PAE. As the DC voltage increases, the output power increases fairly linearly indicating that the amplifier has a constant gain. The efficiency also increases approximately linearly until it reaches the maximum at the optimum 28 volt DC supply voltage.

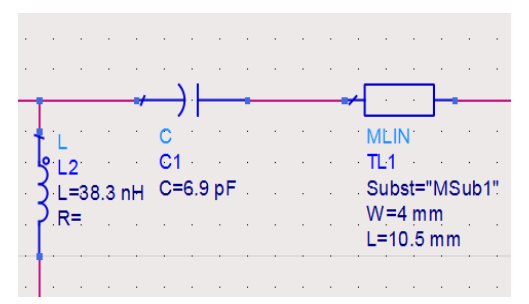

(a)

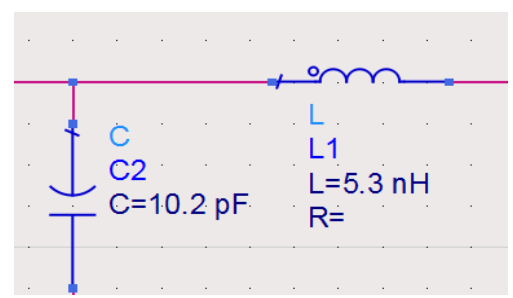

(b)

Figure 2. (a) Input matching network; (b) Output matching network.

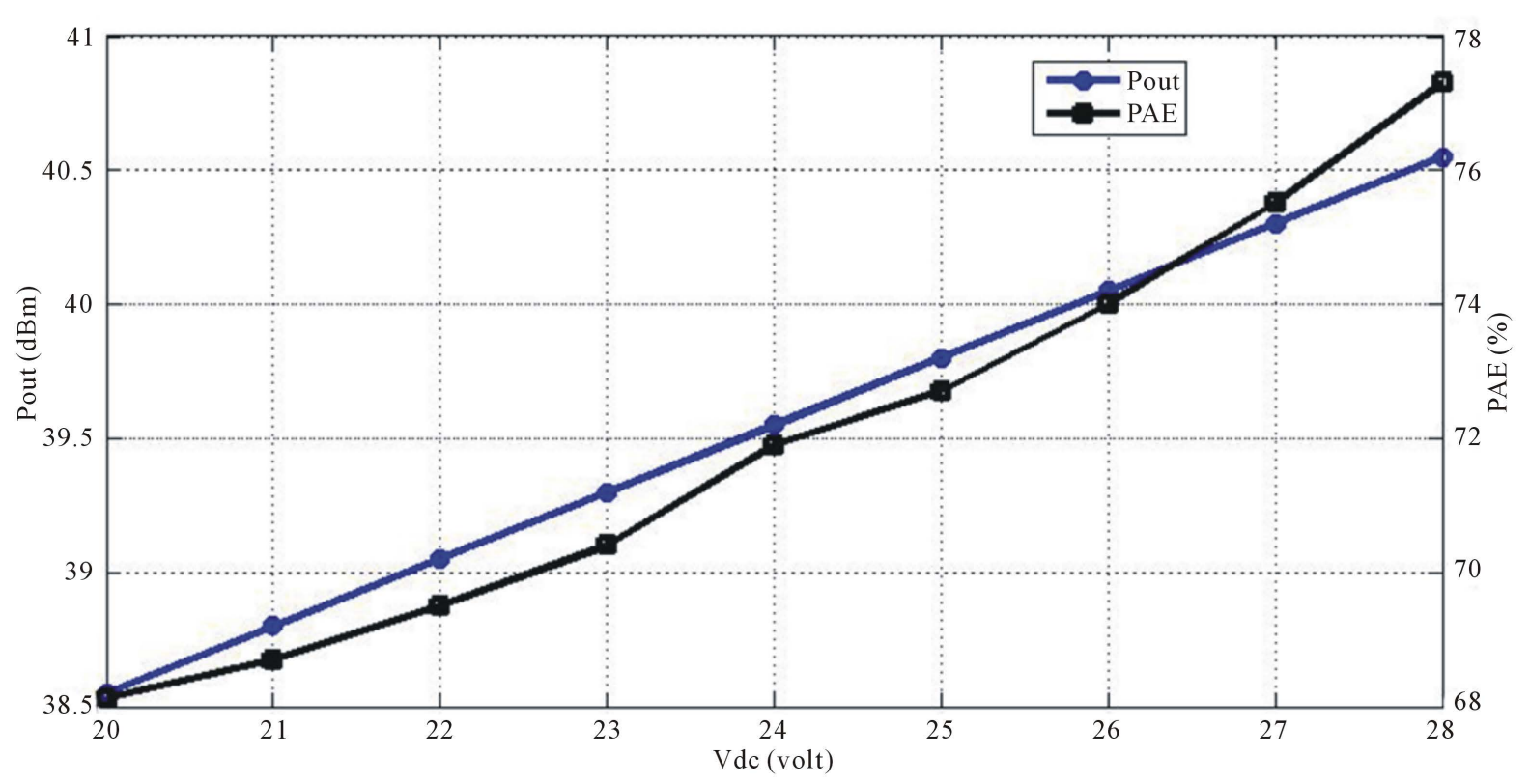

Figure 3. Output power and PAE versus DC voltage in the class AB amplifier.

Table 2. Final results of the class AB amplifier.

\begin{tabular}{cccccc}
\hline Input power $(\mathrm{dBm})$ & DC power $(\mathrm{W})$ & Output power $(\mathrm{dBm})$ & Gain $(\mathrm{dB})$ & PAE $(\%)$ & Drain efficiency $(\%)$ \\
\hline 18.943 & 14.62 & 40.593 & 21.65 & 77.78 \\
\hline
\end{tabular}


The simulated reflection coefficient plots of the class $A B$ amplifier are given in Figure 4. The amplifier is simulated via ADS and AWR software. It is clearly shown that this amplifier can operate from $650 \mathrm{MHz}$ to 910 $\mathrm{MHz}$, thus it is suitable for applications in the TV band. It is observed that the simulation results of the two software tools follow the same pattern with little difference.

The percentage bandwidth or the fractional bandwidth which is a measure of how wideband the amplifier is, is given by this equation:

$$
\% B W=(f 2-f 1) /((f 1+f 2) / 2)
$$

The percentage bandwidth of this amplifier is $33.33 \%$.

The power gain plot of the class $\mathrm{AB}$ amplifier is given in Figure 5. It is clearly shown that this amplifier has a good power gain above $19 \mathrm{~dB}$ for the operation frequency bandwidth.

\subsection{Doherty Power Amplifier}

As mentioned earlier, the Doherty amplifier procedure can be attained using two amplifiers with a class-AB biased carrier amplifier cell and a class-C biased peak amplifier cell with respective input matching network and output matching network. The two power amplifiers are connected at the output through a quarter-wave transmission line.

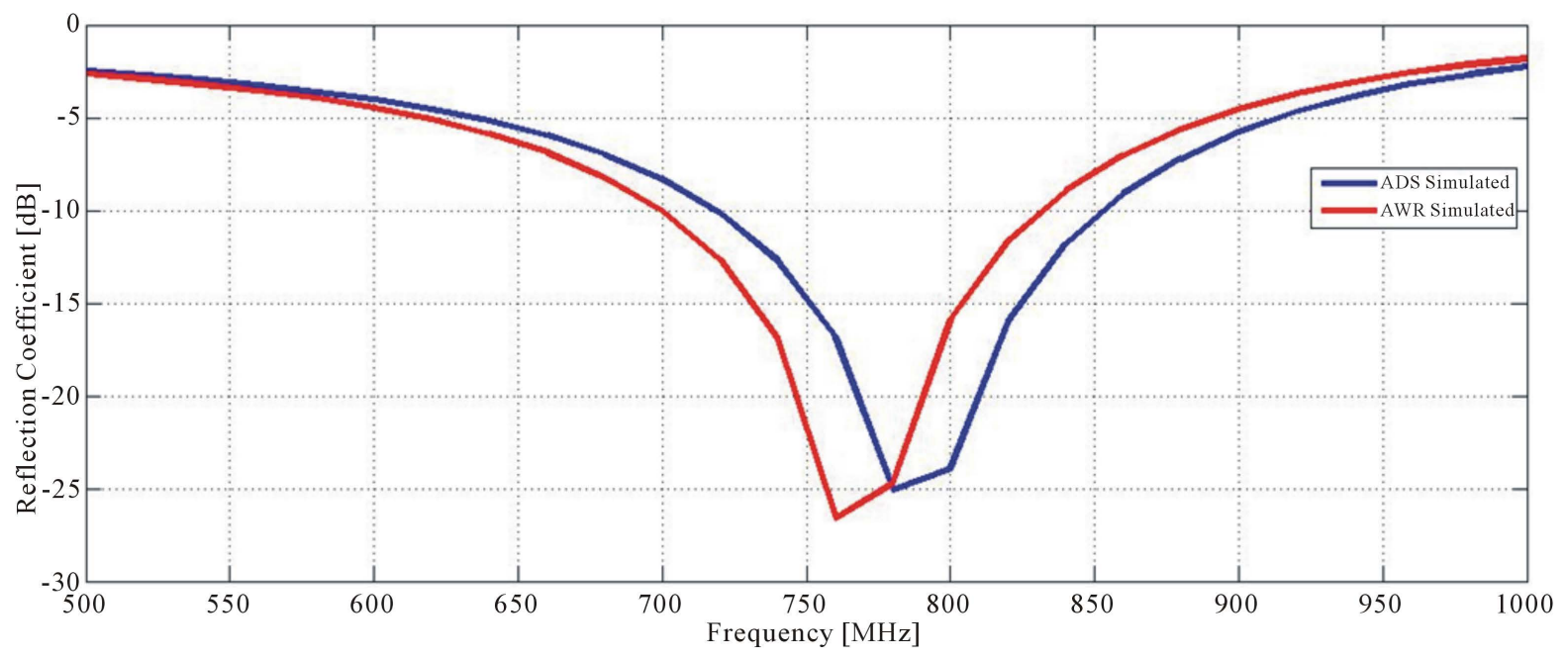

Figure 4. Simulated (ADS and AWR) reflection coefficient plot of the class AB amplifier.

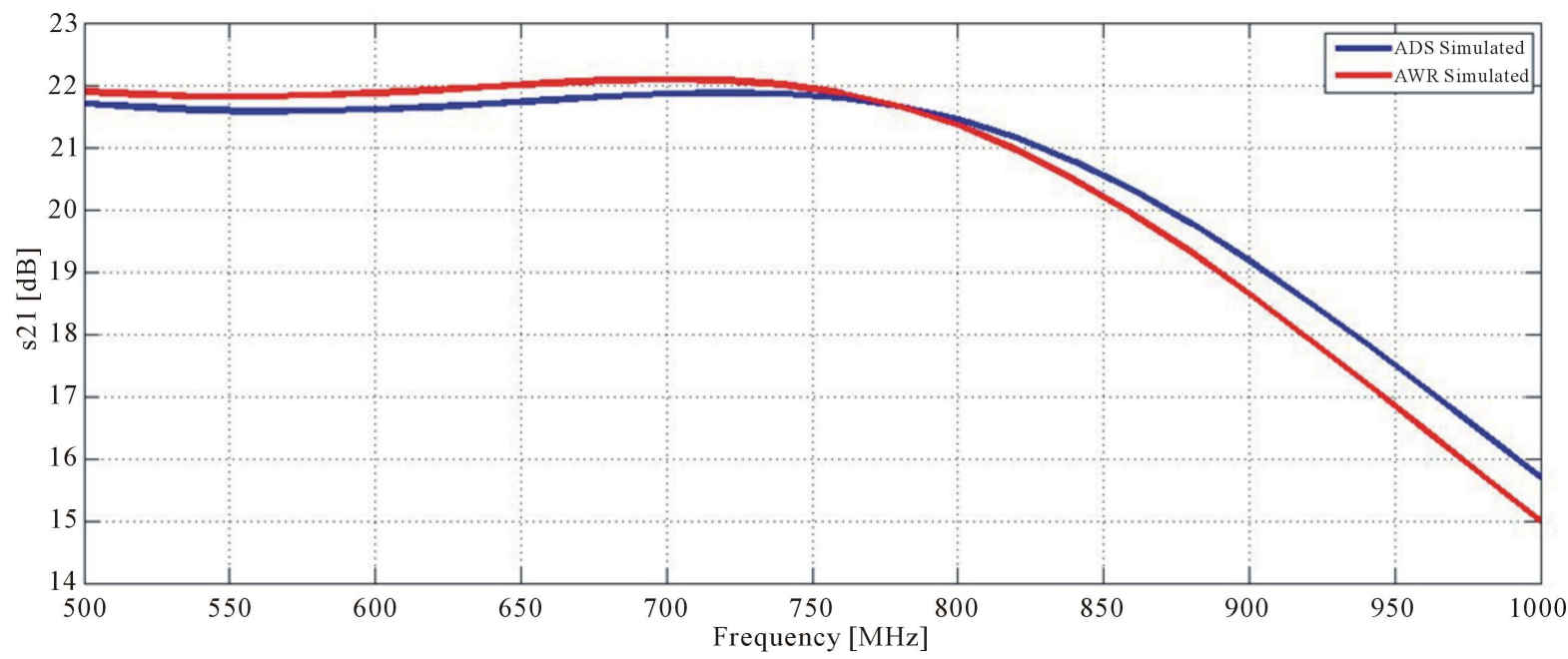

Figure 5. Simulated (ADS and AWR) power gain plot of the class AB amplifier. 
Hereby is a detailed procedure used in designing a Doherty power amplifier followed with a flow chart, shown in Figure 6, which describes this procedure briefly.

Steps for Design of Doherty Amplifier:

1) The active devices (such as transistors) employed in the DPA are specified. They must operate on a wide range of frequencies and have an adequate output power.

2) These active devices are fully matched to ensure maximum power transfer, so input and output matching networks are implemented in the main and the peak amplifiers.

3) Stability of the main and the peak amplifiers are investigated to make sure the two amplifiers are unconditionally stable.

4) The two amplifiers are connected to each other following the well-known Doherty circuit with two transmission lines serving as impedance transformers in order to achieve the main load modulation.

5) An input power divider is inserted before the two amplifiers in order to split the power between the main and the peak amplifiers.

6) The DPA is then matched to attain maximum power transfer, so input and output matching circuits are designed for this purpose.

7) The stability of the DPA is examined and updated so that the DPA is unconditionally stable.

8) Finally, the circuit is fabricated on a primarily specified substrate.

The DPA configuration used in this paper is the well-known conventional AB-C scheme as shown in Figure 7 , with a class $A B$ amplifier used as a main stage and a class $C$ amplifier used as a peak stage.

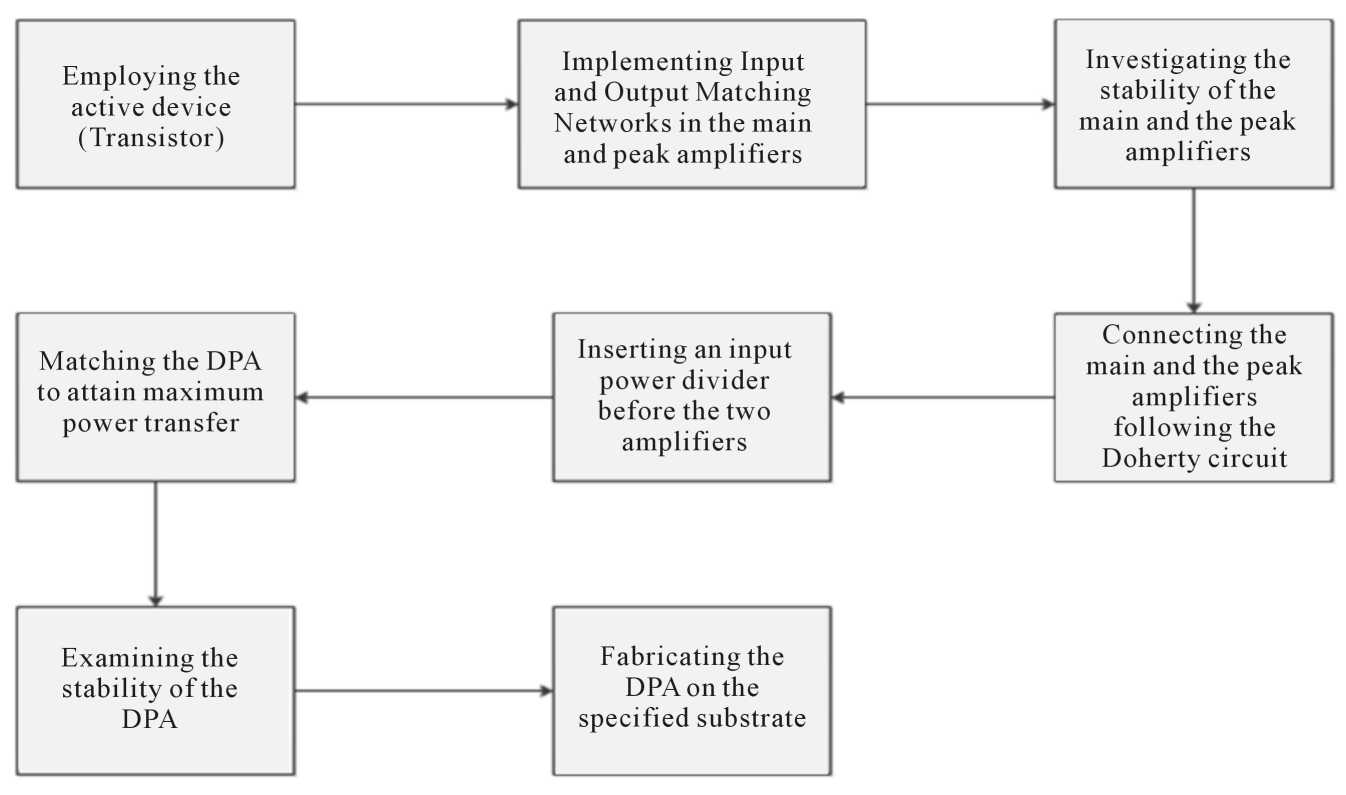

Figure 6. A flow chart of the steps of designing a Doherty power amplifier.

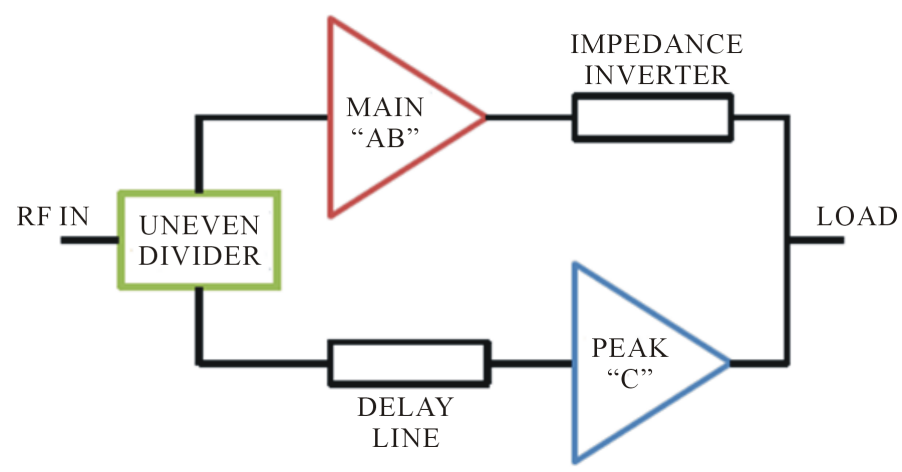

Figure 7. Block scheme of the designed AB-C DPA. 
In our design, the previously designed class $\mathrm{AB}$ amplifier was used as the main amplifier. Hence, to complete the Doherty design, a class $\mathrm{C}$ amplifier is necessary as a peak amplifier. The schematic of this proposed class $\mathrm{C}$ amplifier is shown in Figure 8. The active device employed in the $\mathrm{C}$ amplifier is the ADL5602, an InGaP HBT with a single supply voltage of $5 \mathrm{~V}$. This transistor offers high dynamic range and low noise figure in addition to that it is stable over frequency, temperature, and power supply. The amplifier circuit was simulated at $800 \mathrm{MHz}$ using ADS with a supply voltage Vsup $=5 \mathrm{~V}$ and with input and output matching networks realized by passive elements as well as bias networks. The substrate parameters were set according to Rogers RO3203 substrate with dielectric constant $\varepsilon_{r}=3.02$ and thickness $\mathrm{h}=1.6 \mathrm{~mm}$, in which the amplifier is designed on.

Figure 9 shows the simulated circuit schematic of the proposed AB-C DPA, where the main and the peak amplifiers are as mentioned before. The amplifier is simulated in both software, ADS and AWR. A broadband $90^{\circ}$ hybrid coupler was used at the input in order to split the signals between the carrier and the peak amplifying paths. The electrical lengths of the delay and the impedance inverter microstrip lines were optimized to ensure maximum power transfer, maximum efficiency, and adequate output power.

After carrying out the stability test of the Doherty amplifier, it is found that the device is unconditionally stable in the frequency range of interest. The stability parameters, $\Delta$, and the rollet factor $k$ are calculated and found to be:

$$
\begin{aligned}
& \Delta=0.43 \angle-61.48^{\circ} \\
& k=1.4
\end{aligned}
$$

In order to maintain maximum power transfer, matching circuits are designed at the input and the output of the transistor. These circuits are accomplished by using passive networks and microstrip transmission lines as shown in Figure 10.

Once the amplifier matching is complete, the final signal scattering parameters at $800 \mathrm{MHz}$ were simulated and found to be:
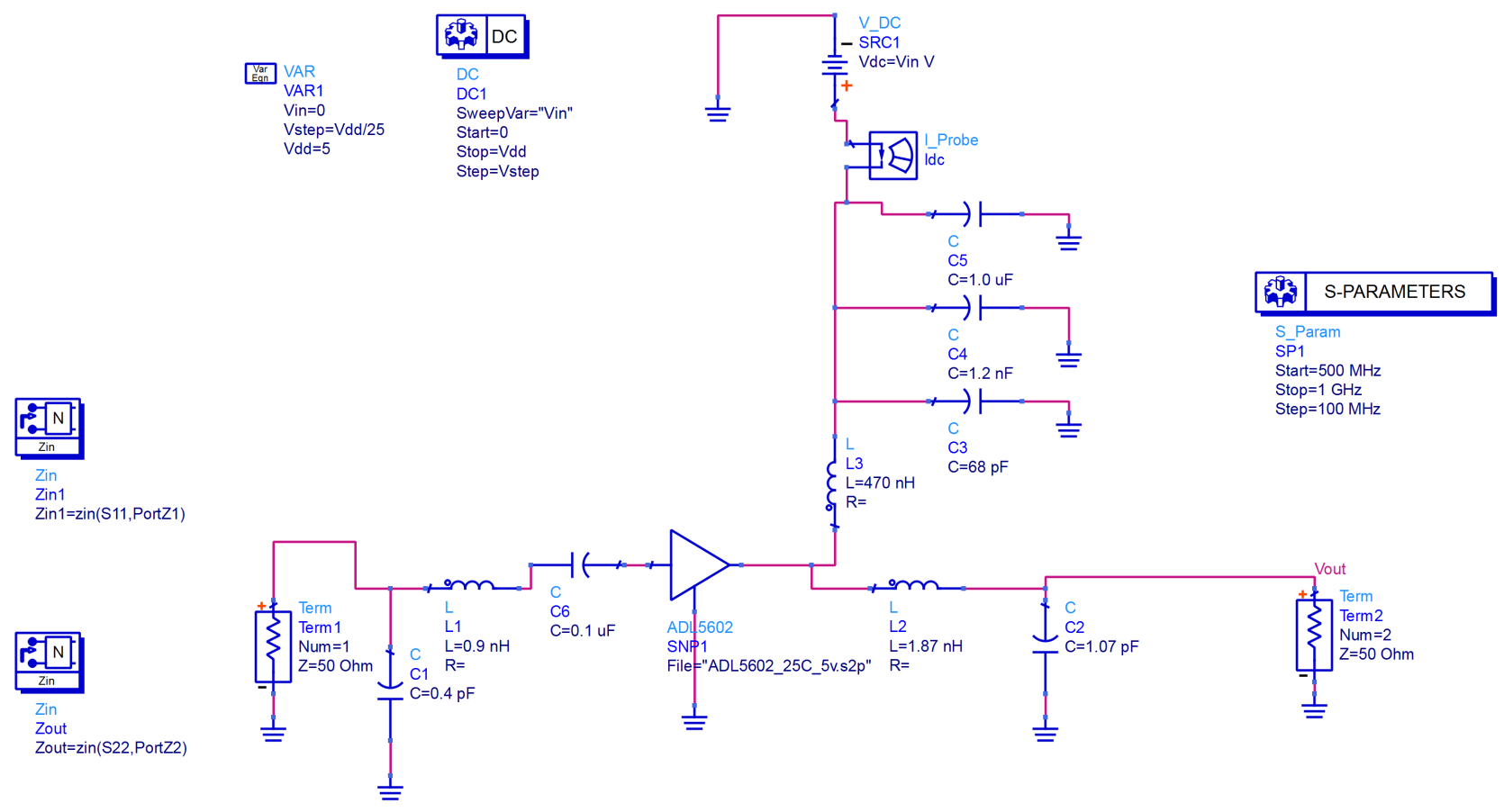

$$
\begin{aligned}
& \mathrm{S} 11=0.198 \angle-99.259^{\circ} \\
& \mathrm{S} 12=0.035 \angle-55.086^{\circ} \\
& \mathrm{S} 21=11.407 \angle 94.509^{\circ} \\
& \mathrm{S} 22=0.175 \angle-90.038^{\circ}
\end{aligned}
$$

Figure 8. Schematic of the $\mathrm{C}$ power amplifier with bias and matching circuits. 


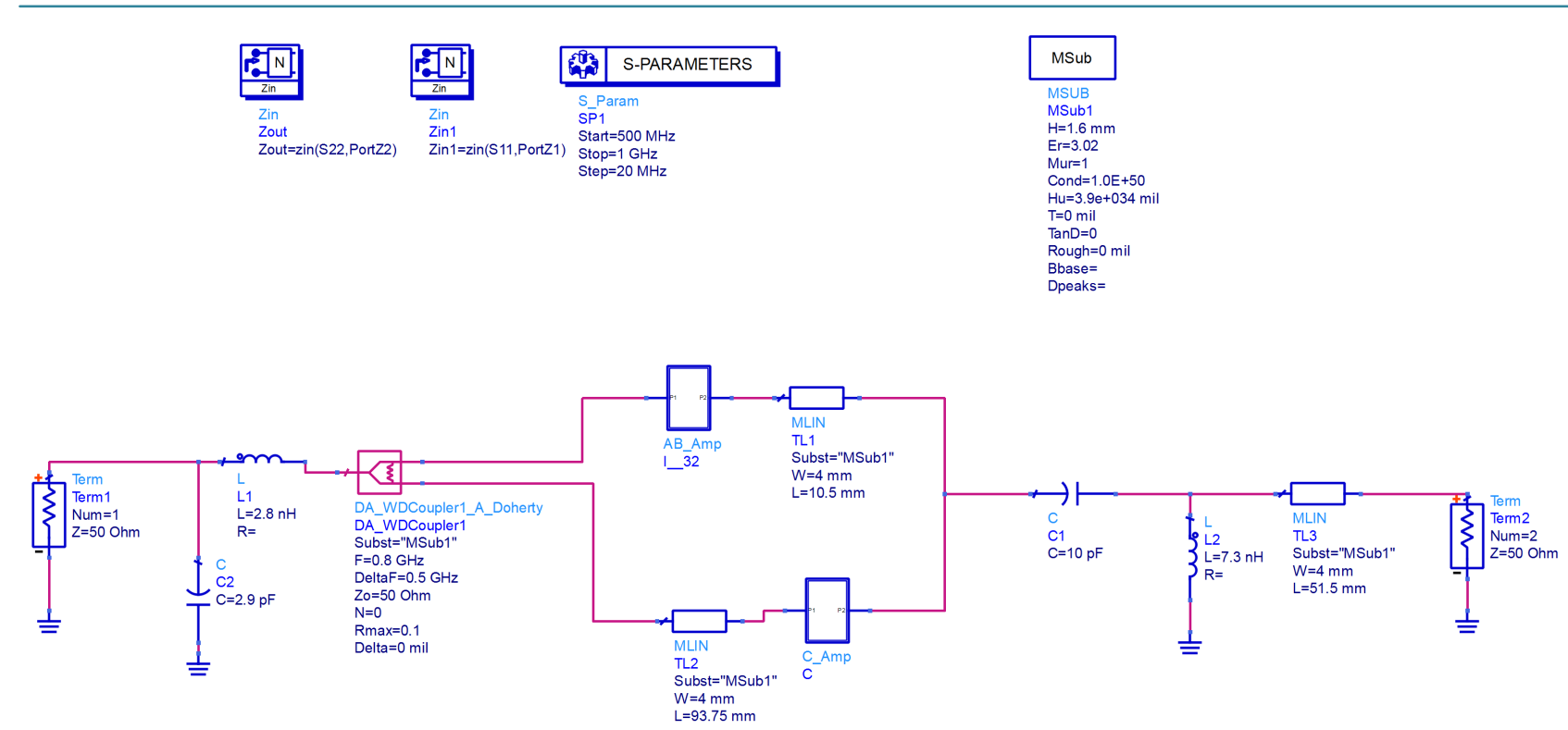

(a)

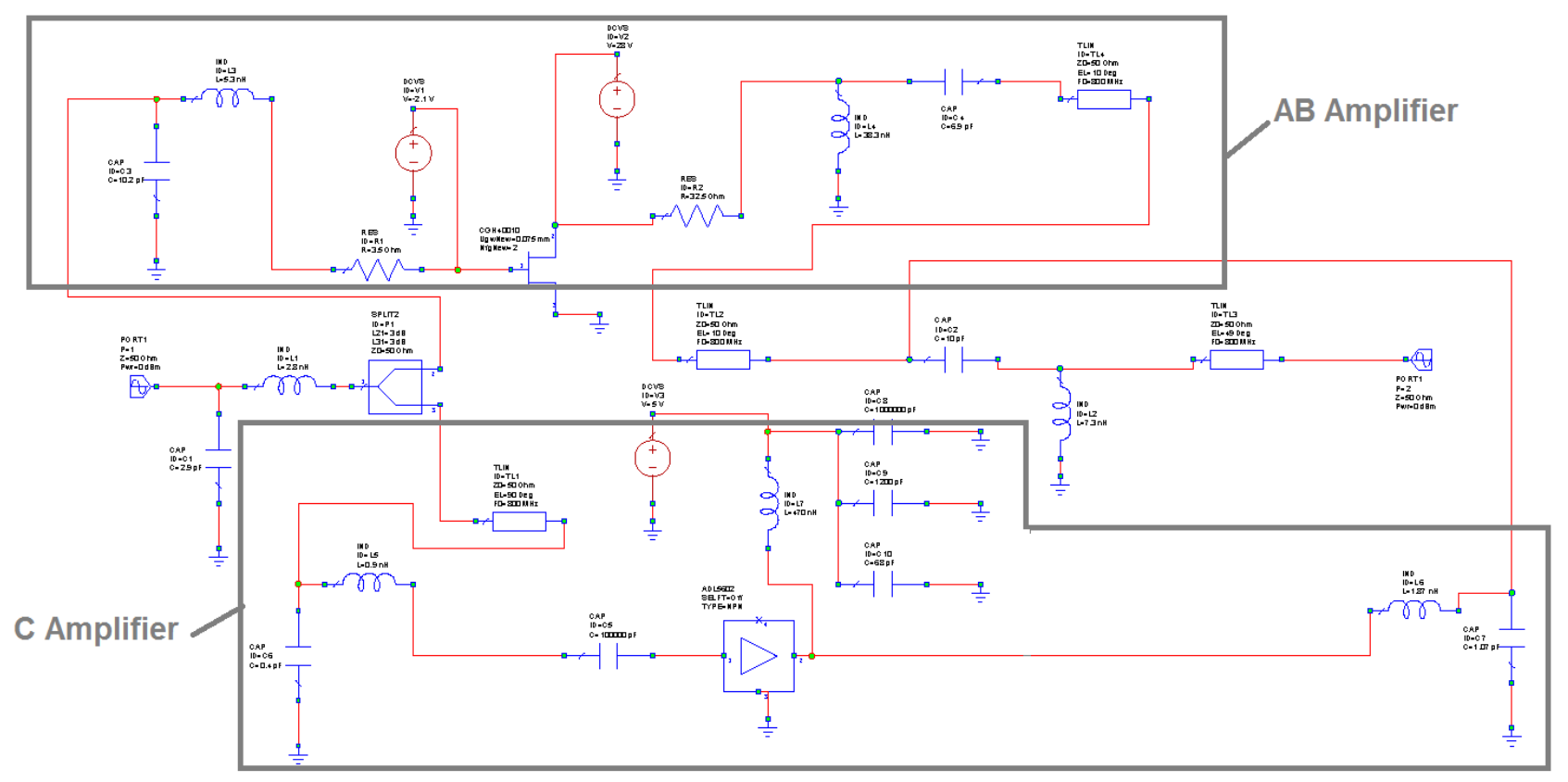

(b)

Figure 9. Schematic of the Doherty power amplifier with matching circuits. (a) In ADS; (b) In AWR.

By feeding the Doherty amplifier with an optimum $800 \mathrm{MHz}, 21.45 \mathrm{dBm}$ input power, the output power of the Doherty amplifier circuit was found to be $42.77 \mathrm{dBm}$ and the gain is $21.32 \mathrm{~dB}$. The DC power is simulated and found to be: Pdc $=22.92 \mathrm{~W}$. Thus, the power added efficiency (PAE) is around $81.94 \%$. All the results are summarized in Table 3. It is clearly shown that the efficiency of the DPA is higher than that of the class AB power amplifier. This is due to the Doherty principle implemented in this design and to the electrical lengths of the delay and impedance inverter transmission line previously mentioned.

Figure 11 shows the output power sweep curve as well as the corresponding PAE. As the DC voltage increases, the output power increases fairly linearly indicating that the amplifier has a constant gain. The efficiency also increases approximately linearly until it reaches the maximum at the optimum 28 volt DC supply voltage. 


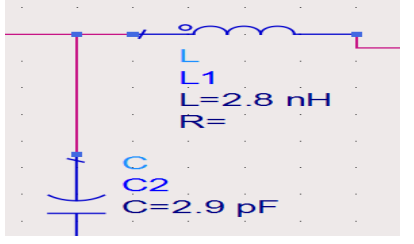

(a)

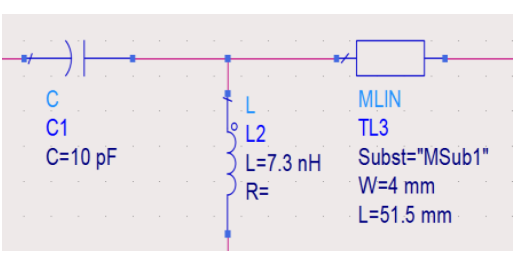

(b)

Figure 10. (a) Input matching network; (b) Output matching network.

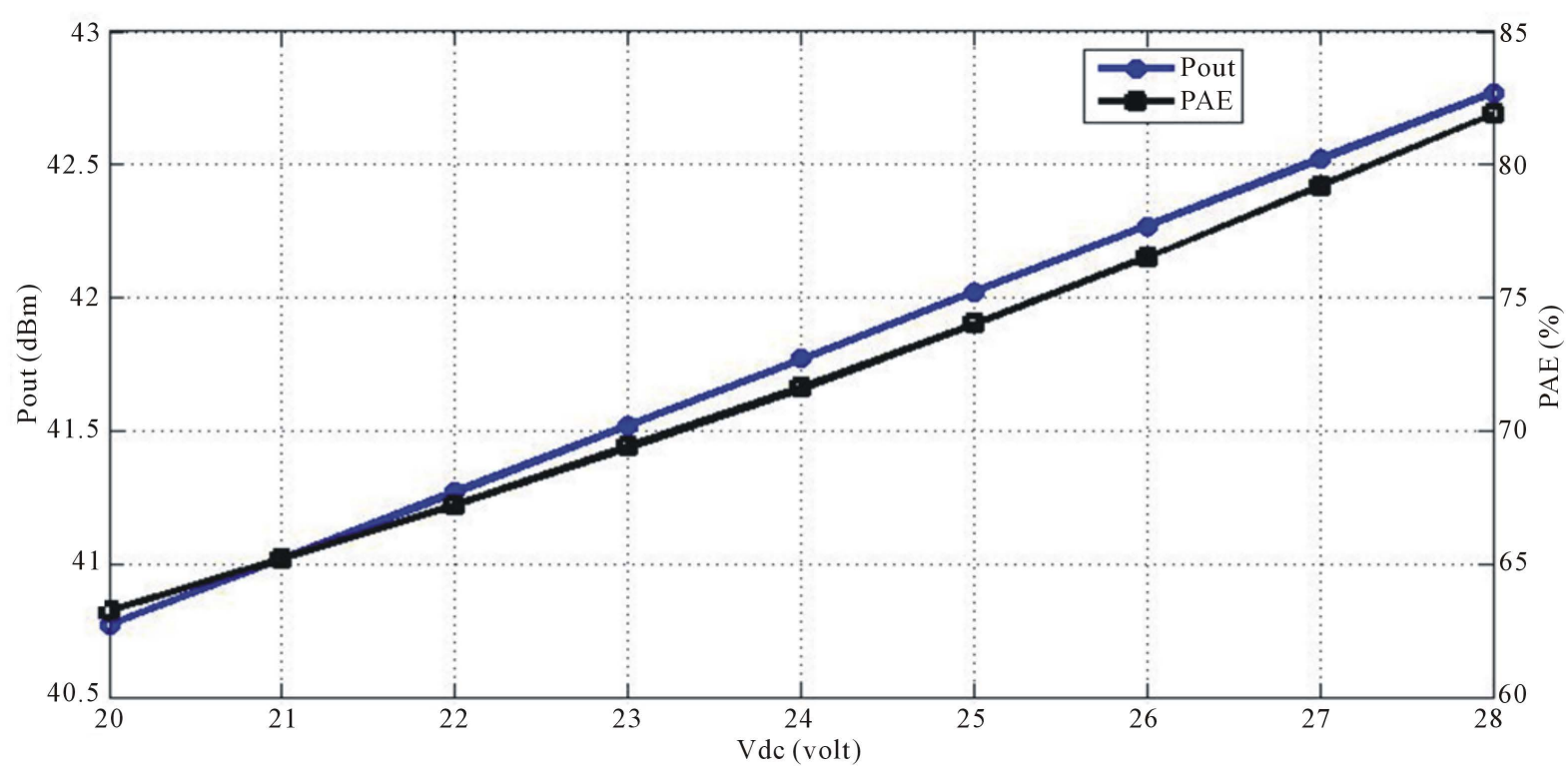

Figure 11. Output power and PAE versus DC voltage in the DPA.

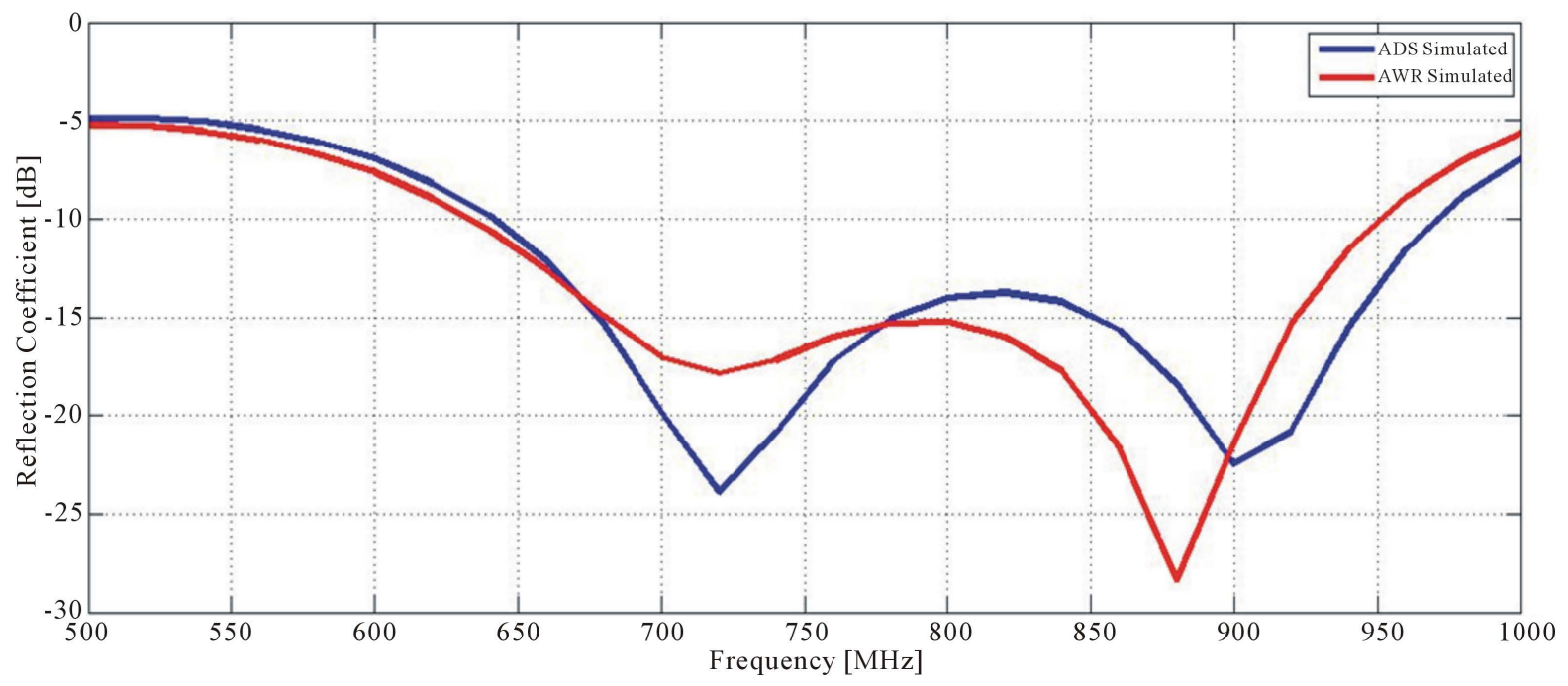

Figure 12. Simulated (ADS and AWR) reflection coefficient plot of the DPA.

Table 3. Final results of the Doherty power amplifier.

\begin{tabular}{|c|c|c|c|c|c|}
\hline Input power (dBm) & DC power $(\mathrm{W})$ & Output power (dBm) & Gain (dB) & PAE (\%) & Drain efficiency (\%) \\
\hline 21.45 & 22.92 & 42.77 & 21.32 & 81.94 & 82.55 \\
\hline
\end{tabular}




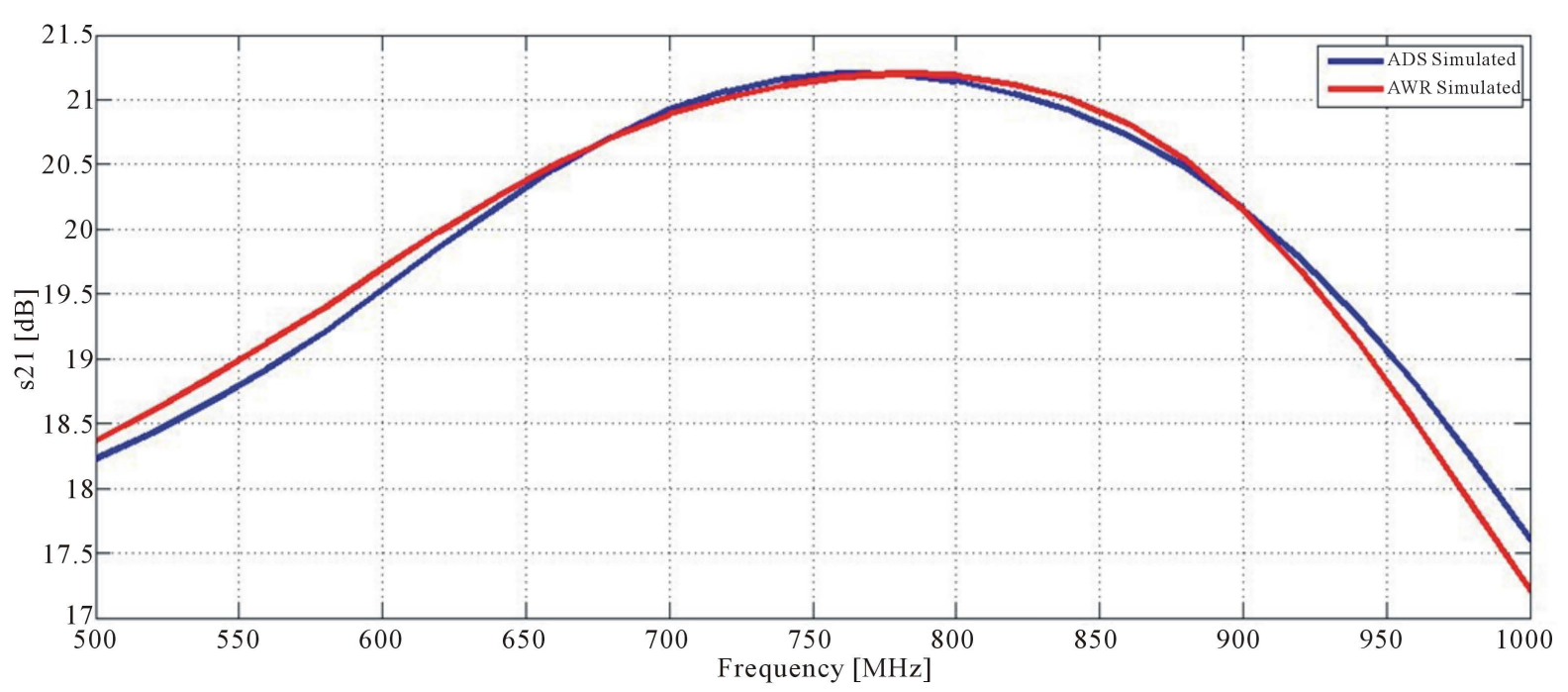

Figure 13. Simulated (ADS and AWR) power gain plot of the DPA.

The reflection coefficient of the DPA is given in Figure 12. It is clearly shown that this amplifier can operate from $550 \mathrm{MHz}$ to $1000 \mathrm{MHz}$, thus it is suitable for all applications in the TV band.

Another advantage of the proposed Doherty amplifier rather than the efficiency increment is that the bandwidth has increased, and the proposed DPA can be suitable for more applications in the TV band. The percentage bandwidth of this DPA is $58.06 \%$ which is higher than that of the class AB power amplifier and higher than Doherty power amplifiers previously designed such as in [13], [15], and [17].

The power gain of the Doherty power amplifier is given in Figure 13. It is clearly shown that this amplifier has a good power gain above $20 \mathrm{~dB}$ for the operation frequency bandwidth.

\section{Conclusion}

A class AB power amplifier and a high efficiency Doherty one were implemented for the TV band applications. A class $A B$ power amplifier was first designed using a commercial GaN HEMT from Cree Incorporation. The Doherty amplifier has been implemented, using the previous class $A B$ amplifier as the main amplifier and a proposed class $\mathrm{C}$ amplifier as the peak one. This proposed DPA attained a high power-added-efficiency of 81.94\%, a $42.77 \mathrm{dBm}$ output power, an associated gain of $21.32 \mathrm{~dB}$, and an operating frequency bandwidth between 550 and $1000 \mathrm{MHz}$ which made it a convenient power amplifier that could be used in TV band applications.

\section{Acknowledgements}

The authors would like to thank the Lebanese National Council for Scientific Research for supporting this research work.

\section{References}

[1] Grebennikov (2005) RF Microwave Power Amplifier Design. McGraw-Hill, New York.

[2] Doherty, W.H. (1936) A New High Efficiency Power Amplifier for Modulated Waves. Proceedings of IRE, 24, 11631182. http://dx.doi.org/10.1109/JRPROC.1936.228468

[3] Ahn, G., Kim, M., Park, H. and Jung, S. (2007) Design of a High-Efficiency and High-Power Inverted Doherty Amplifier. IEEE Transactions on Microwave Theory and Techniques, 55, 1105-1111. http://dx.doi.org/10.1109/TMTT.2007.896807

[4] Bumman, K., Jangheon, K., Ildu, K. and Jeonghyeon, C. (2006) The Doherty Power Amplifier. IEEE Microwave Magazine, 7, 42-50. http://dx.doi.org/10.1109/MW-M.2006.247914

[5] Choi, H.-J., Lim, J.-S., Jeong, Y.-C. and Kim, C.-D. (2006) Doherty Amplifier Using Load Modulation and Phase Compensation DGS Microstrip Line. 36th European Microwave Conference, Manchester, 10-15 September 2006, 352- 
355. http://dx.doi.org/10.1109/eumc.2006.281346

[6] Jung, J., Kim, U., Jeon, J., Kim, J., Kang, K. and Kwon, Y. (2005) A New “Series-Type” Doherty Amplifier for Miniaturization. IEEE Radio Frequency Integrated Circuits (RFIC) Symposium, 12-14 June 2005, 259-262.

[7] Raab, F.H. (1987) Efficiency of Doherty RF Power-Amplifier Systems. IEEE Transactions on Broadcasting, BC-33, 77-83. http://dx.doi.org/10.1109/TBC.1987.266625

[8] Kim, J., Cha, J., Kim, I. and Kim, B. (2005) Optimum Operation of Asymmetrical-Cells-Based Linear Doherty Power Amplifier-Uneven Power Drive and Power Matching. IEEE Transactions on Microwave Theory and Techniques, 53, 1802-1809. http://dx.doi.org/10.1109/TMTT.2005.847073

[9] Kim, J., Moon, J., Woo, Y.Y., Hong, S., Kim, I., Kim, J. and Kim, B. (2008) Analysis of a Fully Matched Saturated Doherty Amplifier with Excellent Efficiency. IEEE Transaction on Microwaves Theory and Techniques, 56, 328-338. http://dx.doi.org/10.1109/TMTT.2007.914361

[10] Lee, Y., Lee, M. and Jeong, Y. (2008) Unequal-Cells-Based GaN HEMT Doherty Amplifier with an Extended Efficiency Range. IEEE Microwave and Wireless Components Letters, 18, 536-538. http://dx.doi.org/10.1109/LMWC.2008.2001015

[11] Markos, Z., Colantonio, P., Giannini, F., Giofrè, R., Imbimbo, M. and Kompa, G. (2007) A 6 W Uneven Doherty Power Amplifier in GaN Technology. Proceedings of 37th European Microwave Conference, Munich, October 2007, 1097-1100.

[12] Steinbeiser, C., Landon, T., Suckling, C., Nelson, J., Delaney, J., Hitt, J., Witkowski, L., Burgin, G., Hajji, R. and Krutko, O. (2008) 250 W HVHBT Doherty with 57\% WCDMA Efficiency Linearized to -55 dBc for 2c11 $6.5 \mathrm{~dB}$ PAR. IEEE Journal of Solid-State Circuits, 43, 2218-2228. http://dx.doi.org/10.1109/JSSC.2008.2004456

[13] Kang, D., Kim, D., Cho, Y., Park, B., Kim, J. and Kim, B. (2011) Design of Bandwidth-Enhanced Doherty Power Amplifiers for Handset Applications. IEEE Transactions on Microwave Theory and Techniques, 59, 3474-3483. http://dx.doi.org/10.1109/TMTT.2011.2171042

[14] Bathich, K., Markos, A.Z. and Boeck, G. (2011) Frequency Response Analysis and Bandwidth Extension of the Doherty Amplifier. IEEE Transactions on Microwave Theory and Techniques, 59, 934-944. http://dx.doi.org/10.1109/TMTT.2010.2098040

[15] Bathich, K. and Boeck, G. (2012) Wideband Harmonically-Tuned GaN Doherty Power Amplifier. 2012 IEEE MTT-S International Microwave Symposium Digest (MTT), Montreal, 17-22 June 2012, 1-3. http://dx.doi.org/10.1109/MWSYM.2012.6259385

[16] Sun, G. and Jansen, R.H. (2012) Broadband Doherty Power Amplifier via Real Frequency Technique. IEEE Transactions on Microwave Theory and Techniques, 60, 99-111. http://dx.doi.org/10.1109/TMTT.2011.2175237

[17] Qureshi, J.H., Li, N., Neo, W.C.E., van Rijs, F., Blednov, I. and de Vreede, L.C.N. (2010) A Wide-Band 20 W LMOS Doherty Power Amplifier. 2010 IEEE MTT-S International Microwave Symposium Digest (MTT), Anaheim, 23-28 May 2010, 1504-1507. http://dx.doi.org/10.1109/MWSYM.2010.5517561 